\section{Detoxifying plant pathogens}

Leaf scald disease of sugarcane is caused by the pathogenic microbe, Xanthomonas albilineans. Toxins produced by the microbe block chloroplast development and have been implicated in the transition from latent to acute symptomatic disease. Zhang et al. (p. 1021) have generated transgenic sugarcane plants expressing a detoxification enzyme, AlbD, and showed that the gene could confer resistance to both disease symptoms and pathogen multiplication in the host.

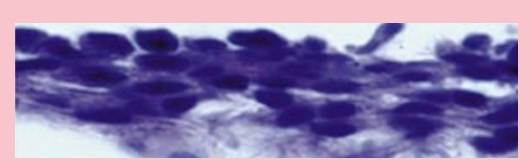

As the bodybuilders at the local gym will tell you, mechanical stress is great for building muscles. Now on page 979, Mooney and colleagues show that this advice applies to building muscle by tissue engineering. They found that by applying cyclic mechanical strain on smooth muscle tissues, they could stimulate production of elastin and collagen, yielding engineered muscle with improved tensile strength and cell alignment.

\section{Mass spec sees double}

Though a powerful strategy for the qualitative analysis of proteins and peptides, mass spectrometry has been less useful for the comparison of individual protein levels between samples. In this issue, Aebersold and coworkers get around this problem using isotopically labeled affinity tags (see p. 994 and Research News p. 954). The "heavy" and "light" tags are bound to peptides from two separate samples, allowing their levels to be easily compared directly within the same mass spectrum.

\section{A neuritogenic synthetic peptide}

On page 1000, Rønn et al. describe a synthetic peptide that can modulate the activity of the neural cell adhesion molecule (NCAM), which plays a key role in neural development, regeneration, and learning. The peptide, identified from a combinatorial library of synthetic peptides, was shown to bind to the Ig module of NCAM, thereby stimulating with high potency a signal transduction cascade leading to neurite outgrowth.

Research Briefs written by Natalie DeWitt and Robert Frederickson.

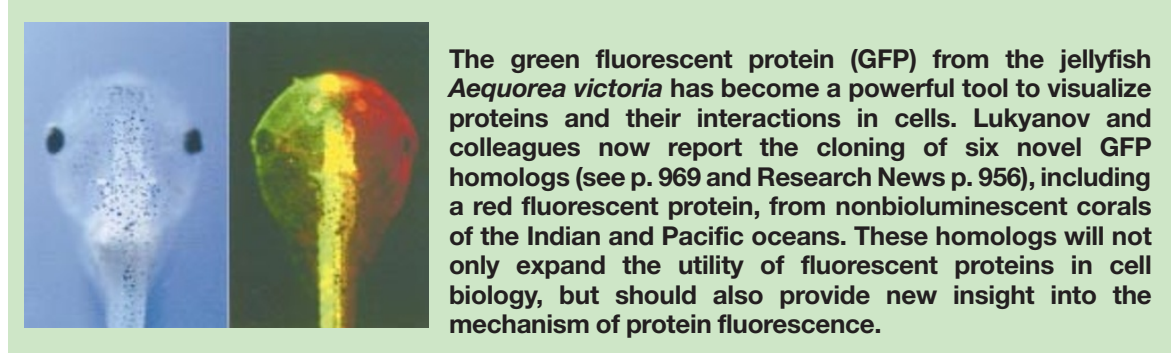

\section{Zapping ocular angiogenesis}

Growth of new blood vessels is a process underlying a number of ocular disorders. On page 984, Birchler et al. describe a way of selectively delivering therapeutics to these blood vessels, using a phage display-derived antibody that specifically recognizes tissues undergoing angiogenesis. As demonstrated in this study, the antibody can deliver photoactive compounds to new blood vessels, for diagnostics of ocular pathologies or targeted ablation of diseased tissue.

\section{Just one word-plastics}

Plastics produced by bacterial fermentation are degradable in many different environments and comprised of renewable resources, making them a "green" alternative to their petroleum-based cousins. A major barrier to commercialization, however, is their high cost. Producing these biopolymers in genetically engineered plants has long been viewed as a way of reducing these costs, but progress has been slow. Now in this issue, Gruys and colleagues describe synthesis of a particularly useful plastic in plants, by engineering four bacterial genes to modify two different metabolic pathways (see p. 1011 and Research News p. 960 ).

DNA chips are usually made by lightdirected oligonucleotide synthesis, with photolithographic masks used to control the addition of nucleotides to the growing chains. A drawback to this technology is the large number of masks needed for each different chip, which makes this technology costly and limits its availability. On page 974 , Sussman and colleagues describe a way around this bottleneck: a maskless array synthesizer (MAS) that uses a digital micromirror array to form computer-generated virtual masks. Developing MAS into a benchtop format could be the next step, making microarrays more versatile and widely available (see also p.953).

\section{SPOTting differences in peptide reactivity}

The reaction of reducing sugars with proteins leads to the formation of advanced glycation end products (AGEs), which are implicated in the pathology of a number of age-related diseases. In this issue, Münch et al. (p. 1006) make use of a combinatorial dipeptide SPOT library synthesized on paper sheets to assess the relative reactivity of amino acid side chains and the $\mathrm{N}$ terminal amino group toward sugars and preformed AGEs. Their approach should be useful in the search for drugs to prevent glycation.

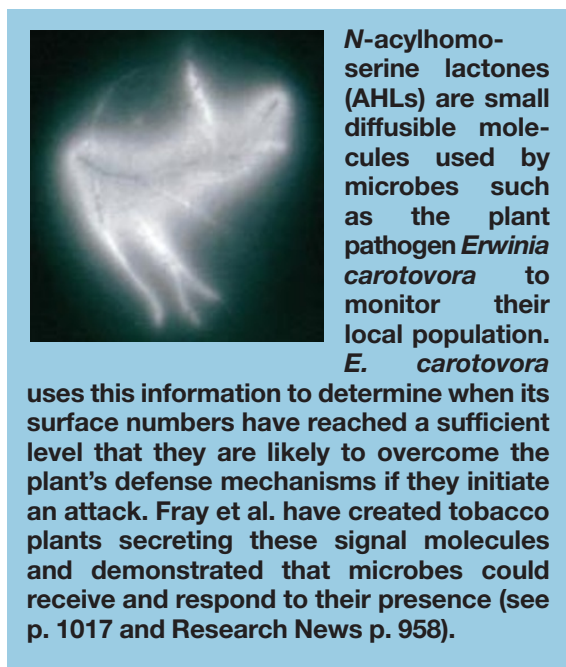

\section{Gene repair redoubles its efforts}

Culver et al. (p. 989) have devised a new oligonucleotide repair strategy for mutation correction in cells with improved precision and efficiency. They use a bifunctional repair oligonucleotide made up of both a heteroduplex-forming repair domain (RD) and a separate triplex-forming domain (TFD) to anchor the oligonucleotide synergistically. Their system corrected point mutations in both the $\mathrm{p} 53$ and human adenosine deaminase genes in human cells. 